\title{
Protective immunity induced by Eimeria common antigen 14-3-3 against Eimeria tenella, Eimeria acervulina and Eimeria maxima
}

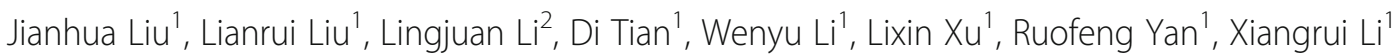 \\ and Xiaokai Song ${ }^{1 *}$ (i)
}

\begin{abstract}
Background: Avian coccidiosis is often caused by co-infection with several species of Eimeria worldwide. Developing a multivalent vaccine with an antigen common to multiple Eimeria species is a promising strategy for controlling clinical common co-infection of Eimeria. In the previous study, 14-3-3 was identified as one of the immunogenic common antigen in E. tenella, E. acervulina and E. maxima. The aim of the present study was to evaluate the immunogenicity and protective efficacy of Ea14-3-3 in the form of DNA vaccine against infection with three species of Eimeria both individually and simultaneously.

Results: After vaccination with pVAX-Ea14-3-3, the Ea14-3-3 gene was transcribed and expressed in the injected muscles. Vaccination with pVAX-Ea14-3-3 significantly increased the proportion of CD4 ${ }^{+}$and $C D 8^{+}$T lymphocytes and produced a strong IgY response in immunized chickens. Similarly, pVAX-Ea14-3-3 stimulated the chicken's splenocytes to produce high levels of Th1-type (IFN- $\gamma$, IL-2) and Th2-type (IL-4) cytokines. The vaccine-induced immune response was responsible to increase weight gain, decreased the oocyst output, and alleviated enteric lesions significantly in immunized chickens as compared to control group, in addition to induce moderate anti-coccidial index (ACI).

Conclusion: These results indicate that Ea14-3-3 is highly immunogenic and capable to induce significant immune responses. Furthermore, Ea14-3-3 antigen can provide effective protection against infection with Eimeria tenella, Eimeria acervulina, Eimeria maxima both individually and in combination with three Eimeria species. Significant outcomes of our study provide an effective candidate antigen for developing a multivalent Eimeria vaccine against mixed infection with various Eimeria species under natural conditions.
\end{abstract}

Keywords: Chicken coccidian, Common antigen 14-3-3, Mixed infection, Immunogenicity, DNA vaccine

\section{Background}

Avian coccidiosis is one of the most widespread and economically detrimental diseases in the poultry industry. It causes severe damage to the host intestine, resulting in impaired feed intake, increased mortality and increased susceptibility to other disease agents [1,2]. It has been estimated that the annual loss due to coccidiosis exceeds $\$ 3$ billion USD globally [3]. Primarily, control of avian

\footnotetext{
* Correspondence: songxiaokai@njau.edu.cn

${ }^{1} \mathrm{MOE}$ Joint International Research Laboratory of Animal Health and Food

Safety, College of Veterinary Medicine, Nanjing Agricultural University,

Nanjing 210095, People's Republic of China

Full list of author information is available at the end of the article
}

coccidiosis is based on the use of anti-coccidial drugs and live vaccines [1]. Although these two approaches have been generally effective for controlling the disease, the drawbacks of these two approaches, such as drug resistance and residue, high cost and lack of uniformity in vaccination have prompted the search for new generation vaccines including subunit vaccines and DNA vaccines [3-6]. The causes of clinical coccidiosis in the intensive farming are infection by various Eimeria species [3, 7]. The species of Eimeria tenella, E. acervulina and E. maxima are commonly found in all commercial birds $[1,8,9]$. Thus, applied vaccines should contain protective antigens

(c) The Author(s). 2018 Open Access This article is distributed under the terms of the Creative Commons Attribution 4.0 International License (http://creativecommons.org/licenses/by/4.0/), which permits unrestricted use, distribution, and reproduction in any medium, provided you give appropriate credit to the original author(s) and the source, provide a link to the Creative Commons license, and indicate if changes were made. The Creative Commons Public Domain Dedication waiver (http://creativecommons.org/publicdomain/zero/1.0/) applies to the data made available in this article, unless otherwise stated. 
common to relevant species and confer effective protection against mixed infection with Eimeria species [10].

Several antigens common to Eimeria have been reported previously. Talebi et al. [11] found an immunogenic protein $(45 \mathrm{kDa})$ among five Eimeria species. While, Sasai et al. [12] observed a common antigen present on conoid of six chicken's Eimeria sporozoites. Additionally, Constantinoiu et al. [13] reported highly conserved apical antigens among subjected Eimeria species. However, the reported common antigens were not well identified by sequencing and their protective efficacies have not been evaluated. In the earlier study of our lab, Ea14-3-3 antigen was identified as one of the common immunodominant antigens from E. tenella, E. acervulina and E. maxima [14]. It has been documented that 14-3-3 proteins are involved in many patho-physiological and cellular immune processes by triggering or interfering with the activity of specific protein associates [15]. In apicomplexan parasites, the 14-3-3 protein plays a vital role in parasite invasion, molecular and biological processes with immuno-protective responses [16-20]. Moreover, in E. tenella, 14-3-3 antigen was proven to interact with telomerase activity and involved in the process of coccidia development [17].

The 14-3-3 proteins have been showed an immunogenic response, and able to stimulate the host immune activity in some parasites. Schechtman et al. [21] reported that the 14-3-3 antigen of Schistosoma mansoni influenced the significant humoral and cellular responses and induced moderate protection against challenged infection. In Toxoplasma gondii, the 14-3-3 protein induced effective immune responses in BALB/c mice and was suggested as a novel DNA vaccine candidate against toxoplasmosis [20]. Besides that, 14-3-3 antigens in Trichinella spiralis and Echinococcus were documented to be highly immunogenic and described as promising vaccine targets against infections [22, 23]. Therefore, $14-3-3$ protein may be potential vaccine candidates against these parasites.

In the current study, the immunogenicity and protective efficacy of Ea14-3-3 against E. tenella, E. acervulina and E. maxima was further investigated. Results of this study may provide an effective candidate antigen for developing a multivalent Eimeria vaccine against mixed infection with multiple species of Eimeria under natural conditions.

\section{Results}

\section{Sequence analysis and eukaryotic expression plasmid construction of Ea14-3-3 gene}

The ORF of Ea14-3-3 gene was cloned into pMD18-T and confirmed by sequencing. The ORF of Ea14-3-3 gene is composed of 837 nucleotides with predicted molecular weights of $31.77 \mathrm{kDa}$ (Additional file 1: Figure S1). Sequence analysis showed that Ea14-3-3 has similarity of $100 \%$ in nucleotides and amino acid sequences with the genes in NCBI (XM_013394831). Ea14-3-3 has a high amino acid similarity of more than $94 \%$ among the four chicken coccidia species (Additional file 2: Table S1). The constructed pVAX-Ea14-3-3 plasmid was confirmed by endonuclease cleavage and sequence analysis. Endonuclease cleavage with BamH I /Xho I produced a band of about $837 \mathrm{bp}$, which is equal to the size of the inserted gene Ea14-3-3 (Additional file 3: Figure S2 lane 2). The fragment was extracted from the gel and sequenced. The sequence analysis revealed that the inserted gene has $100 \%$ similarity of nucleotides and amino acid sequences with the Ea14-3-3 gene.

\section{Ea14-3-3 was transcribed and expressed in the injected site of chickens}

RT-PCR assay was employed with the specific primers for Ea14-3-3 to detect transcriptions of the Ea14-3-3 gene in the injected muscles. Agarose electrophoresis showed a band of approximately $837 \mathrm{bp}$ from the muscle injected with pVAX-Ea14-3-3 (Fig. 1a, lane 1). No specific DNA bands were detected in pVAX1 injected and non-injected control sample (Fig. 1a, lane 2 and 3). These results indicate that Ea14-3-3 was transcribed in the injected site muscles of chickens.

Western blot assay was employed with primary antibody of anti-E. acervulina chicken sera to detect the expressed proteins. As shown in Fig. 1b, in pVAX-Ea14-3-3 injected muscle anti-E. acervulina chicken sera reacted with a protein band of approximately $32 \mathrm{kDa}$ (Fig. 1b, lane 1). No specific band was detected in non-injected control and
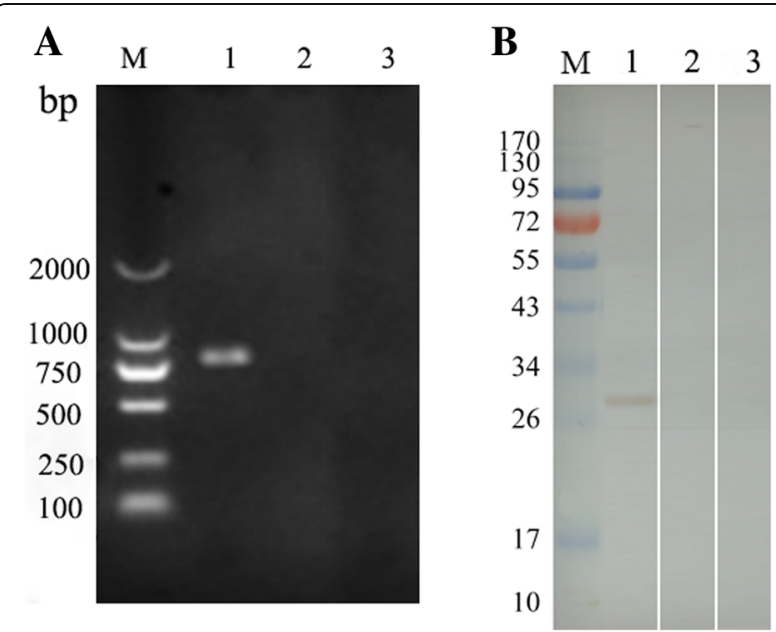

Fig. 1 Transcription and expression detection of Ea14-3-3 gene in injected muscle. a RT-PCR analysis of chicken muscles injected with pVAX-Ea14-3-3 M: DL2000 Marker. Lane 1: RT-PCR product of chicken muscles injected with pVAX-Ea14-3-3. Lane 2: non-injected muscle. Lane 3: pVAX1 plasmid injected muscle. b Western blot analysis of chicken muscles injected with pVax-Ea14-3-3; M: Protein molecular weight marker. Lane 1: Western blot analysis of chicken muscles injected with pVAV-Ea14-3-3. Lane 2: non-injected muscle. Lane 3: pVAX1 plasmid injected muscle 
vector control samples (Fig. 1b, lane 2 and 3). These results indicate that Ea14-3-3 was expressed in the injected site muscles of chickens.

\section{Ea14-3-3 induced significant cellular immune responses} in chickens

The proportions of $\mathrm{CD}^{+} / \mathrm{CD}^{+}$and $\mathrm{CD}^{+} / \mathrm{CD}^{+}$splenic $\mathrm{T}$ lymphocytes from vaccinated chickens were determined by flow cytometry assay. As per depicted in Fig. 2, 1 week after the primary and booster dose of vaccination, the proportions of $\mathrm{CD}^{+} / \mathrm{CD}^{+}$and $\mathrm{CD}^{+} / \mathrm{CD}^{+}$splenic $\mathrm{T}$ lymphocytes from vaccinated chickens were significantly higher than those from PBS and pVAX1 empty plasmid control chickens $(p<0.05)$. No significant difference was observed between the pVAX1 and PBS control chickens $(p>0.05)$. The results demonstrated that Ea14-3-3 effectively promoted the $\mathrm{T}$ lymphocyte responses in chickens. The mRNA levels of IFN- $\gamma$, IL-2, IL-4, TNFSF15, IL-17D and TGF- 34 cytokines from vaccinated chickens were determined by qPCR. As shown in Fig. 3, 1 week after the primary and booster dose of immunization, the production of IFN- $\gamma$, IL-2, IL-4 TNFSF15, IL-17D and TGF- $\beta 4$ was significantly increased in pVAX-Ea14-3-3-vaccinated chickens as compared to pVAX1-vaccinated and PBS control chickens $(p<0.05)$. No significant difference was observed between pVAX1 and PBS control chickens $(p>0.05)$. These results indicated that Ea14-3-3 effectively promoted the production of cytokines in chickens.

In a word, the Eimeria common antigen 14-3-3 effectively induced the secretion of cytokines in chickens.

\section{Ea14-3-3 induced a significant serum antibody response} in chickens

The indirect ELISA method was employed to determine the antibody response induced by Ea14-3-3. As shown in Fig. 4, from 1 week to 6 weeks post-booster vaccination,

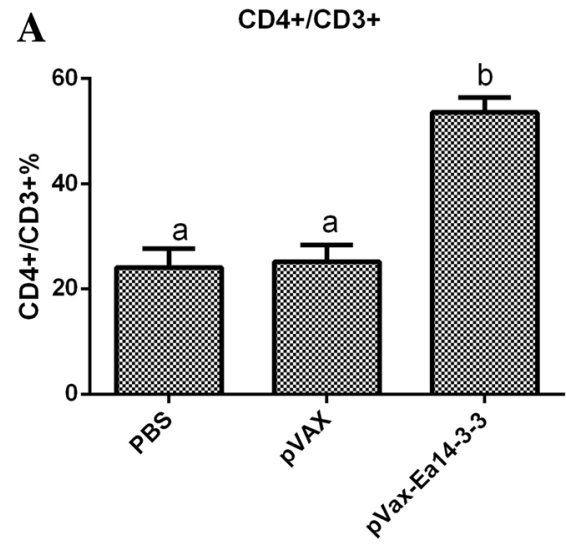

One week after the primary vaccination

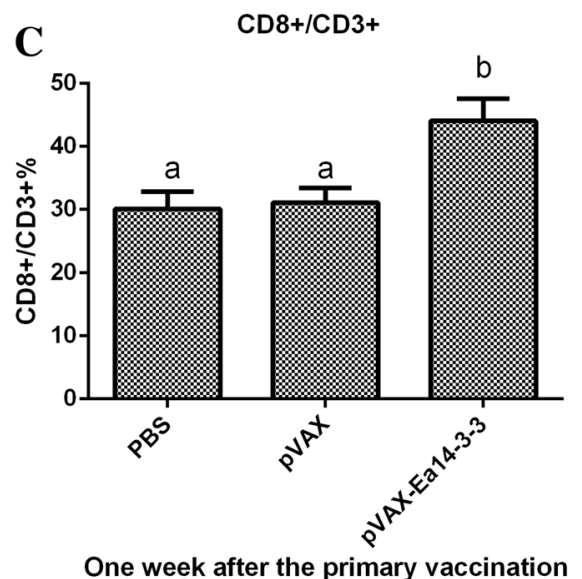

B

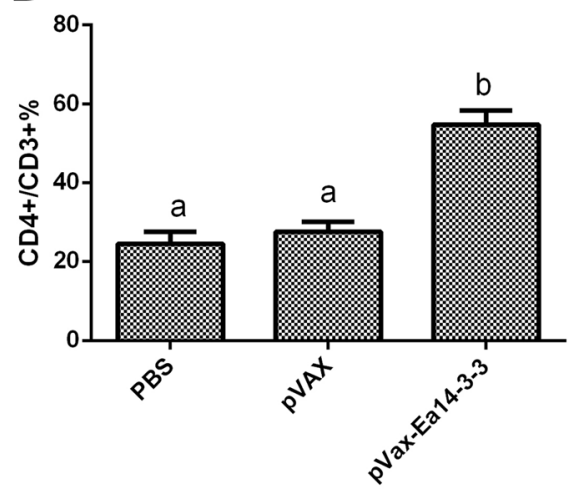

One week after the booster vaccination
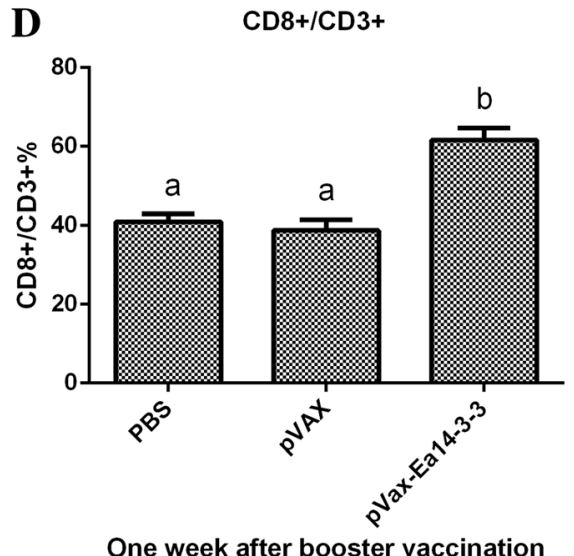

Fig. 2 Changes of proportion of $\mathrm{CD}^{+} / \mathrm{CD}^{+}$and $\mathrm{CD}^{+} / \mathrm{CD}^{+} T$ cells in spleens of the chickens vaccinated with pVAX-Ea14-3-3. (a) Proportion of $\mathrm{CD}^{+} / \mathrm{CD}^{+} \mathrm{T}$ cells in spleens of the chickens 1 week after the primary vaccination; (b) proportion of $\mathrm{CD} 4^{+} / \mathrm{CD}^{+} \mathrm{T}$ cells in spleens of the chickens 1 week after the booster vaccination; (c) proportion of $\mathrm{CD}^{+} / \mathrm{CD}^{+} \mathrm{T}$ cells in spleens of the chickens 1 week after the primary vaccination; (d) proportion of $\mathrm{CD}^{+} / \mathrm{CD}^{+} \mathrm{T}$ cells in spleens of the chickens 1 week after the booster vaccination; significant difference $(p<0.05)$ between numbers with different letters; non-significant difference $(p>0.05)$ between numbers with the same letter 

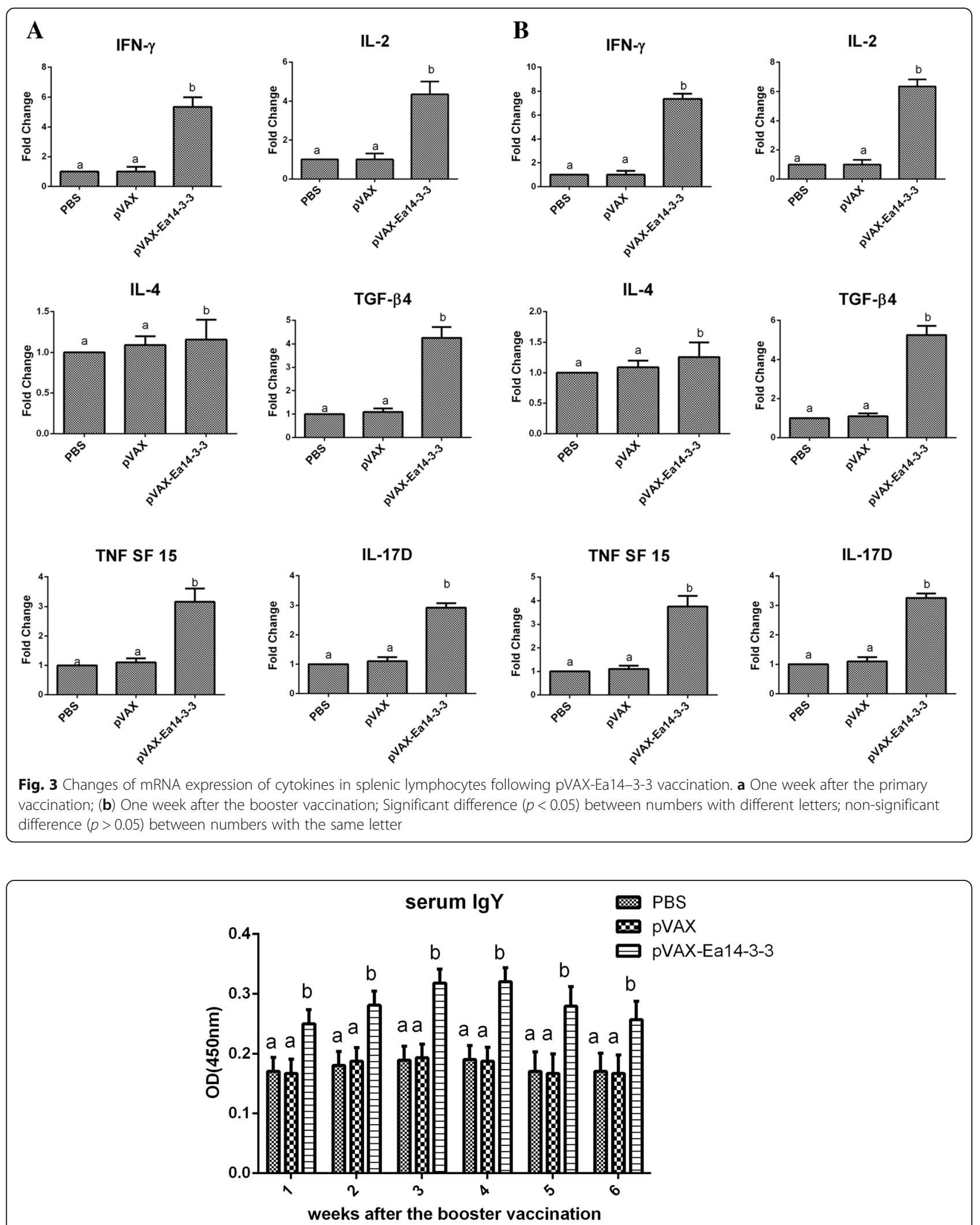

Fig. 4 Serum specific IgY levels in chickens following the recombinant plasmid pVAX-Ea-14-3-3 vaccination. At weeks 1, 2, 3, 4, 5 and 6 post-second immunization, blood was collected by cardiac puncture and antibody levels were determined by ELISA. Each bar represents the mean $\pm S . D$. $(N=5)$. Values with different superscripts in the same differ significantly $(P<0.05)$ 
the induced antibody titers of all pVAX-Ea14-3-3 vaccinated groups were significantly higher as compared to the PBS and pVAX1 empty plasmid control groups. No significant difference was observed between the pVAX1 and PBS control group $(p>0.05)$. The antibody titers of vaccinated groups increased slowly from the first week to the third week post-booster immunization, peaked at the fourth week, and then decreased gradually PBS and pVAX1 empty plasmid groups were not detected any specific antibodies. The results showed that Ea14-3-3 significantly induced significant serum antibody response in chickens.

Ea14-3-3 induced effective protection against $E$. tenella, $E$. acervulina and E. maxima

To evaluate the protective efficacy of pVAX-Ea14-3-3, groups of chickens were challenged with $E$. tenella, $E$. acervulina, E. maxima or mixed oocysts of the three Eimeria species. As shown in Table 1, vaccination with pVAX-Ea14-3-3 significantly increased the body weight gains, decreased the oocyst output, and alleviated the enteric lesions of vaccinated groups as compared to PBS control and pVAX1 control groups $(p<0.05)$. No significant difference was observed between the pVAX1 and PBS control group $(p>0.05)$. The vaccination resulted in ACIs of 171.31(E. acervulina), 161.03(E. maxima), 178.29 (E. tenella) and 170.92 (mixed Eimeria). This result indicates that Ea14-3-3 provided effective protections against challenge with E. tenella, E. acervulina, E. maxima and mixed infection of the three Eimeria species.

\section{Discussion}

Avian coccidiosis, one of the most potential destructive diseases in birds, is caused by several Eimeria species under natural conditions. Thus an ideal field Eimeria vaccine should provide effective protection against co-infection with mixed Eimeria species [2, 9, 24-26]. Common antigens shared among Eimeria species are extremely promising for the development of multivalent Eimeria vaccines against multiple Eimeria species. Previous studies have revealed such a few common antigens among Eimeria which had serological cross reactions among certain species [13]. For example, Talebi [11] found a few shared proteins between species and at least one protein band $(45 \mathrm{kDa})$ was conserved among the five species. The conserved protein band of all these species could be recognized by chicken anti-E. maxima sera. Sasai et al. [12] identified a common conoid sporozoites antigen among 6 different Eimeria species (E. brunetti, E. maxima, E. mitis, E. necatrix, E. praecox and $E$. tenella) by confocal laser scanning microscopy using a chicken monoclonal antibody (mAb) 6D12-G10 against E. acervulina sporozoite. Constantinoiu et al. [13] analyzed cross-reactivity of five chicken mAb against $E$. acervulina sporozoites using confocal laser immunofluorescence assay and found $\mathrm{mAb} 8 \mathrm{E}-1$ recognized an

Table 1 Protective efficacy of common antigen Ea14-3-3 against challenge with E. tenella, E. acervulina, E. maxima and mixed oocysts of the three Eimeria species

\begin{tabular}{|c|c|c|c|c|c|c|}
\hline Group & $\begin{array}{l}\text { Challenge with } \\
\text { Eimeria spp. }\end{array}$ & $\begin{array}{l}\text { Average body } \\
\text { weight gain (g) }\end{array}$ & $\begin{array}{l}\text { Relative body } \\
\text { weight gain (\%) }\end{array}$ & Mean lesion scores & $\begin{array}{l}\text { Oocyst decrease } \\
\text { ratio (\%) }\end{array}$ & $\mathrm{ACl}$ \\
\hline pVAX-Ea14-3-3 & E. tenella & $53.26 \pm 11.52^{b}$ & 87.49 & $0.42 \pm 0.52^{b}$ & 58.79 & 178.29 \\
\hline pVAX1 control & E. tenella & $35.26 \pm 9.89^{a}$ & 58.00 & $3.59 \pm 0.51^{c}$ & -0.61 & 112.1 \\
\hline Challenged control & E. tenella & $32.58 \pm 10.29^{a}$ & 53.11 & $3.78 \pm 0.67^{c}$ & 0.00 & 105.41 \\
\hline Unchallenged control* & PBS & $61.30 \pm 9.29^{c}$ & 100 & $0 \pm 0^{a}$ & 100 & 200 \\
\hline pVAX-Ea14-3-3 & E. acervulina & $53.96 \pm 12.0^{b}$ & 88.51 & $1.62 \pm 0.54^{b}$ & 77.05 & 171.31 \\
\hline pVAX1 control & E. acervulina & $38.41 \pm 10.93^{\mathrm{a}}$ & 63.20 & $3.01 \pm 0.81^{c}$ & 12.67 & 93.1 \\
\hline Challenged control & E. acervulina & $37.38 \pm 10.27^{a}$ & 62.37 & $3.26 \pm 0.67^{c}$ & 0.00 & 89.77 \\
\hline Unchallenged control* & PBS & $61.30 \pm 8.89^{c}$ & 100 & $0 \pm 0^{a}$ & 100 & 200 \\
\hline pVAX-Ea14-3-3 & E. maxima & $52.96 \pm 11.91^{b}$ & 88.23 & $1.72 \pm 0.64^{b c}$ & 72.04 & 161.03 \\
\hline pVAX1 control & E. maxima & $35.39 \pm 11.52^{\mathrm{a}}$ & 58.62 & $2.56 \pm 0.71^{c}$ & 12.19 & 93.02 \\
\hline Challenged control & E. maxima & $35.18 \pm 12.21^{a}$ & 57.18 & $2.90 \pm 0.67^{c}$ & 0.00 & 88.18 \\
\hline Unchallenged control* & PBS & $61.30 \pm 9.29^{c}$ & 100 & $0 \pm 0^{a}$ & 100 & 200 \\
\hline pVAX-Ea14-3-3 & Mixed oocysts & $52.98 \pm 11.54^{b}$ & 86.82 & $0.59 \pm 0.51^{b}$ & 39.33 & 170.92 \\
\hline pVAX1 control & Mixed oocysts & $35.10 \pm 10.81^{a}$ & 57.57 & $3.58 \pm 0.61^{c}$ & -1.69 & 111.77 \\
\hline Challenged control & Mixed oocysts & $33.08 \pm 11.09^{a}$ & 53.77 & $3.70 \pm 0.57^{c}$ & 0.00 & 106.77 \\
\hline Unchallenged control* & PBS & $61.30 \pm 9.29^{c}$ & 100 & $0 \pm 0^{a}$ & 100 & 200 \\
\hline
\end{tabular}

Significant difference $(p<0.05)$ between numbers with different letters. No significant difference $(p>0.05)$ between numbers with the same letter *unchallenged control was shared among the groups challenged with different Eimeria species

Mixed oocysts: mixed oocysts of E. tenella, E. acervulina and E. maxima 
apical tip molecule present on all chicken Eimeria sporozoites. However, these above studies merely revealed molecular weight or fluorescence localization of the common antigens. They did not further identify the specific common antigen by individual sequencing. In our previous study, at least 5 specific Eimeria common immunodominant antigens among three Eimeria species have been identified by immuno-proteomic analysis and LC-MS/MS technique [13]. The 14-3-3, one of the five identified Eimeria common immunogenic antigens is highly conserved among three Eimeria species (Additional file 2: Table S1). In the present observation, we further evaluated immunogenicity and protective efficacy of common antigen of 14-3-3 against challenge with $E$. tenella, E. acervulina and E. maxima. We found that vaccination with the common antigen Ea14-3-3 contributed effective protection not only against individual infection with E. tenella, E. acervulina, or E. maxima, but also against the co-infections with corresponding species. Hence, these findings provide a promising common antigen for developing a vaccine against clinical co-infection by multiple Eimeria species.

Cellular immune responses play a dominant role in the immunity against coccidiosis [27]. In this study, the cellular immune responses induced by $E a-14-3-3$ were assessed. The results showed that the proportions of spleen $\mathrm{T}$ lymphocyte subpopulations of $\mathrm{CD}_{4}^{+}$and $\mathrm{CD} 8^{+}$ were significantly increased. High levels of Th1-type (IFN- $\gamma$, IL-2) and Th2-type (IL-4) cytokines were produced. These vaccine-induced immune responses resulted in effective protections against Eimeria. These results are consistent with other reported findings. Bessay et al. [28] found that E. acervulina infection induced a significant increase in the proportion of $\mathrm{CD}^{+}$and $\mathrm{CD}^{+}$in the duodenal intraepithelial leucocytes (IEL) from day 4 to day 8 post infection (pi). Min et al. [29] found that the ratios of $\mathrm{CD}^{+} / \mathrm{CD}^{+}$and $\mathrm{CD}^{+} / \mathrm{CD}^{+}$ were remarkably increased after immunizing chickens with a pcDNA3-1E vaccine.

Several cytokines have been shown to be involved in immune responses to Eimeria infection [27, 30]. Th1-type cytokines such as IFN- $\gamma$ and IL-2 are responsible for cellular immunity and dominant during Eimeria infection [27, 30]. The cytokine IFN- $\gamma$ has been demonstrated to be important in immuno-regulation in coccidial infections [26, 31]. Recombinant chicken IFN- $\gamma$ could inhibit the intracellular development of $E$. tenella in vitro and reduce oocyst production and body weight loss following $E$. acervulina challenge infection [30, 32]. IL-2 is considered as a potent growth factor for T-cell differentiation, B-cell development and NK-cell activation [30, 33]. IL-2 mRNA transcripts level in the spleen and intestine was significantly enhanced after infections with E. acervulina. IL-2 has been demonstrated to be able to significantly improve the protective effect of recombinant coccidia genes by DNA vaccination $[29,34]$. In this study, the mRNA transcripts level of IFN- $\gamma$ and IL-2 were significantly increased by vaccination with pVAX-Ea14-3-3 (Fig. 3). Furthermore, proportions of $\mathrm{CD}^{+} / \mathrm{CD}^{+}$and $\mathrm{CD}^{+} / \mathrm{CD}^{+} \mathrm{T}$ lymphocytes were significantly increased (Fig. 2). The increasing trend of $\mathrm{CD}^{+} /$ $\mathrm{CD}^{+}$and $\mathrm{CD}^{+} / \mathrm{CD}^{+}{ }^{+} \mathrm{T}$ lymphocytes in accordance with IFN- $\gamma$ and IL-2, indicating that T-cell immune response might be prompted by Th1-type cytokines. IL-4, a typical Th2-type cytokine, is responsible for regulating humoral immunity [35]. In this study, the mRNA transcripts level of IL-4 was significantly increased by vaccination with pVAX-Ea14-3-3 (Fig. 3), which in accordance with high level of antibody response in the vaccinated chickens. IL-17D produced by Th17 cells, participates in the induction of inflammation during protozoan infection. After infection with E. maxima and E. tenella, the expression of IL-17 increased significantly in lymphocytes of the spleen $[36,37]$. In this study, the mRNA transcripts level of IL-17D was significantly increased by vaccination with pVAX-Ea14-3-3 (Fig. 3). TGF- $\beta$ is an anti-inflammatory cytokine that downregulate inflammatory responses and promote repair of damaged mucosal epithelial integrity following injury [30, 38]. After infected with E. acervulina, the mRNA level of TGF- $\beta 4$ in spleen and intestinal epithelial endothelial cells was significantly higher than that in uninfected chickens [39]. In this study, the mRNA level of TGF- $\beta 4$ was significantly increased by vaccination with pVAX-Ea14-3-3. The high level of TGF- $\beta 4$ might help repair the mucous membrane damaged by Eimeria parasites. TNF is able to promote the proliferation and differentiation of IL-2 and IFN- $\gamma$, enhancing the stimulation of antigen on B cells [40]. In vivo experiments revealed that TNFSF15 gene was highly increased following primary infections with Eimeria [41]. In this study, the mRNA level of TNFSF15 was significantly increased following vaccination with pVAX-Ea14-3-3. In short, vaccination with pVAX-Ea14-3-3 induced high level of IFN- $\gamma$, IL-2, IL-4, TNFSF15, IL17D and TGF- $\beta$, playing important roles in immune responses against Eimeria infection.

Although the Ea14-3-3 DNA vaccine provided effective protection against Eimeria, some measures could be taken to improve protection and to make it more practical for use in fields [42]. The protective efficacy of the DNA vaccine could be enhanced by co-injection with plasmids encoding immune stimulating cytokines (IFN- $\gamma$, IL-2) [29, 34, 43]. The sugarcane (Saccharum officinarum L.) bagasse-derived polysaccharides could be used as native immunomodulatory candidate to improve the protective efficacy of the DNA vaccine [44].

In the current study, an optimal vaccination procedure including two injections was used to obtain best efficacy for the DNA vaccine. However, on commercial broiler poultry farms, farmers prefer to administer single 
vaccinations via non-injection delivery routes at day 1 of age. Hence, the vaccination procedure must be further optimized to make vaccination with pVAX-Ea14-3-3 more practical, for example, identify the minimal vaccination age and evaluate the efficacy of a single vaccination and non-injection delivery routes [45].

\section{Conclusion}

The coccidal common antigen of Ea14-3-3 induced significant humoral and cellular immune response against Eimeria infection. Vaccinations with DNA vaccine of Ea14-3-3 had significant ability to induce effective protection against infection of individual Eimeria species $(E$. tenella, E. acervulina and E. maxima), while, also in the mixed infection of these species. Our study indicates that effective common antigen of 14-3-3 could be used in the development of multivalent vaccine against co-infections of multiple Eimeria species in commercial poultry industry.

\section{Methods}

\section{Chicken, parasite and vector}

One-day-old Hy-Line layer chickens were conventionally reared in standardized and sterilized wire cages to prevent intensive contact with any contamination. The birds were given with coccidiostat-free feed and water ad-libitum. Animal experiment was approved by the Institutional Animal Care and Use Committee of Nanjing Agricultural University (approval number: 2012CB120762). Oocysts of E. tenella, E. acervulina and $E$. maxima used in challenged infection were propagated, harvested and sporulated 7 days prior to the challenged infection, using a previously described protocol [46]. The eukaryotic expression vector pVAX1 was purchased from Invitrogen (Carlsbad, California, USA).

\section{Antisera preparation}

Two-week-old chickens were orally inoculated with $1 \times$ $10^{4}$ sporulated oocysts of $E$. acervulina 3-5 times per bird by the interval of 3-days. Negative control birds were inoculated with distilled water. One week post the third inoculation, wing vein blood was collected and determined by ELISA.Forth to fifth dose was given to chickens unless titers of the sera antibody were beyond 1: 64. Serum was stored at $-20{ }^{\circ} \mathrm{C}$ for Western blot analysis, while negative control serum was collected from negative control chickens.

\section{Cloning of the 14-3-3 gene}

Micro glass beads were used to break the E. acervulina sporulated oocysts via whirl mix [46]. Briefly, equal volume of $0.5-\mathrm{mm}$-diameter glass beads and oocysts were mixed in a tube and agitated on a whirl mix with the maximum speed for about $8-10$ bursts of $1 \mathrm{~s}$ each. At the interval of every 4 bursts, supernatant sample was scrutinized by microscopic examination to ensure that most of the oocysts were broken and sporocysts were intact. Subsequently, the sporocysts were recovered from the glass beads by repeated additions of medium. The sporozoites were released from the sporocysts by in vitro excystation with trypsin $0.25 \%(w / v)$ and taurocholic acid $1 \%(\mathrm{w} / \mathrm{v})$ at $41{ }^{\circ} \mathrm{C}$. Finally, the sporozoites were purified over nylon wool and DE-52 cellulose columns according to the manufacturer's instructions [46]. Total RNA was extracted from $E$. acervulina sporozoites using E.Z.N.A. TM Total RNA Kit I (OMEGA, Norcross, Georgia, USA) according to the manufacturer's instructions. Reverse transcription reaction (RT) was performed to produce cDNA with Oligo (dT) as primers [47]. With the complementary DNA (cDNA) as a template, the complete open reading frame (ORF) of the Ea14-3-3 gene (GenBank Accession No. XM_013394831) was amplified by polymerase chain reaction $(\mathrm{PCR})$ using specific primers designed for Ea14-3-3 ORF (BamH I anchored forward primer 5'-CGCGGATCCATGATTGAGGACATCAAGACTCTT -3', Xho I anchored reverse primer 5'-CCGCTCGAG CTACTGCTGCTCAGTAGTAGCTT-3'). The PCR products were cloned into pMD18-T vector (TaKaRa Biotech, Dalian, China) to produce pMD18-T-Ea14-3-3. The resultant plasmid was identified by endonuclease digestion and sequencing. Basic local alignment search tool (BLAST) (http://www.ncbi.nlm.nih.gov/BLAST/) was used to analyze the nucleotide sequence.

\section{Construction of eukaryotic expression plasmid pVAX- \\ Ea14-3-3}

The ORF of Ea14-3-3 was cloned into eukaryotic expression vector pVAX1 to construct pVAX-Ea14-3-3. Briefly, fragments of Ea14-3-3 were excised from the pMD18-T-Ea14-3-3 by BamH I and Xho I digestion and ligated into pVAX1 at the same enzyme sites to construct pVAX-Ea14-3-3. The resulting plasmid was confirmed by endonuclease cleavage and sequence analysis.

\section{Transcription detection of the Ea14-3-3 gene by RT-PCR in chickens}

Two-week-old chickens were vaccinated with $100 \mu \mathrm{g}$ dose of the recombinant plasmid pVAX-Ea14-3-3 through intramuscular injection in the thigh region. While, control group chickens were injected with the pVAX1 vector as described above. A small circle was marked on the injection site and kept clear until the cutting of muscle sample. One week later, injected site muscle sample $(\sim 0.5 \mathrm{~g})$ was excised from each chicken for mRNA extraction. Potential residual plasmids were removed by DNase I (TaKaRa) digestion. RT-PCR was employed with the RNA product as template using the specific primers for Ea14-3-3 ORF. Electrophoresis in $1 \%$ agarose gel was subsequently performed to detect the Ea14-3-3 fragment. Muscles from 
the corresponding site of non-injected chickens were also lacerated as control samples.

\section{Expression detection of Ea14-3-3 gene by Western blot in chickens}

Two-week-old chickens were immunized with $100 \mu \mathrm{g}$ dose of the recombinant plasmid pVAX-Ea14-3-3 or pVAX1 vector as mentioned previously. One week later, a sample of each injected muscles (about $0.5 \mathrm{~g}$ ) was obtained as before, and treated with RIPA lysis buffer $(0.1 \mathrm{~mol} / \mathrm{L}$ phenylmethylsulfonyl fluoride (PMSF), $50 \mathrm{mmol} / \mathrm{L}$ Tris- $\mathrm{HCl}$, $150 \mathrm{mmol} / \mathrm{L} \mathrm{NaCl}, 1 \%$ Nonnidet P-40, 0.1\% SDS) for $3 \mathrm{~h}$. The muscle sample from the corresponding site of each non-injected chicken was used as the control. The samples were centrifuged at 13,000 rpm for $10 \mathrm{~min}$, and the supernatant was collected for Western blot analysis. For detection of expressed proteins, Western blot assay was performed using anti-E. acervulina chicken sera as primary antibody as previously reported method $[48,49]$.

\section{Immunogenicity evaluation of Ea14-3-3 in chickens Experimental design}

Two-week-old chickens were randomly divided into 3 groups of 40 chickens in each group. As described previously, experimental groups of chickens were vaccinated with $100 \mu \mathrm{g}$ of recombinant plasmid pVAX-Ea14-3-3. Vector control group chickens were injected with $100 \mu \mathrm{g}$ of empty pVAX1, and the PBS control group chickens were injected with same volume of sterile PBS ( $\mathrm{pH}$ 7.4). One week later, all chickens were received booster injection. One week after the primary and booster immunizations, 5 chickens from each group were euthanized by cervical dislocation for evaluation of $\mathrm{T}$ lymphocyte sub-populations and cytokines production separately. Blood sera were collected from the rest of 30 chickens in each group for specific antibody determination.

Flow Cytometry analysis of T lymphocyte subpopulations Ea14-3-3 antigen induced changes in T lymphocyte subpopulations were determined using flow cytometry. Spleens from 5 euthanized chickens of each group were collected to evaluate spleen's $\mathrm{T}$ lymphocyte subpopulation proportions of $\mathrm{CD}^{+}$and $\mathrm{CD}^{+}$. The spleens were cut into pieces and gently pushed through a mesh $(250 \mu \mathrm{m}$ pore size). Spleen lymphocyte suspensions were prepared as described previously [50]. The cells $\left(1 \times 10^{6}\right.$ cells $\left./ \mathrm{ml}\right)$ were dually stained with mouse anti-chicken CD3-PE/Cy5, mouse anti-chicken CD8 $\alpha$-FITC, and mouse anti-chicken CD3-PE/Cy5 + mouse anti-chicken CD4-FITC at room temperature in the dark for $30 \mathrm{~min}$. After 3 washes twice PBS by centrifugation $(2000 \mathrm{rpm}$ for 5 min at $4{ }^{\circ} \mathrm{C}$ ), splenocytes population were determined by FACScan flow cytometer and analyzed with Cell Quest software (BD Biosciences, Franklin Lakes, NJ, USA).

\section{Determination of cytokine transcription by quantitative real-time PCR}

Spleen lymphocytes from the vaccinated chickens (five per group) were prepared as previously described [50]. Total RNA was extracted from spleen lymphocytes using an E.Z.N.A. Total RNA Kit Maxi Kit (OMEGA). The cDNA was then generated using RT-PCR. Quantitative real-time PCR (qPCR) was employed to determine IFN- $\gamma$, IL-2, IL-4 TNFSF15, IL-17D and TGF- $\beta 4$ mRNA levels in immunized chickens. The qPCR was carried out with an initial denaturation at $95{ }^{\circ} \mathrm{C}$ for $30 \mathrm{~s}$, followed by 40 cycles at $95^{\circ} \mathrm{C}$ for $10 \mathrm{~s}$, at $60{ }^{\circ} \mathrm{C}$ for $30 \mathrm{~s}$ and followed by a melting curve program at $95^{\circ} \mathrm{Cfor}$ $15 \mathrm{~s}$, at $60^{\circ} \mathrm{Cfor} 15 \mathrm{~s}$, at $95^{\circ} \mathrm{C}$ for $15 \mathrm{~s}$, using an $\mathrm{ABI}$ PRISM 7500 Fast Real-Time PCR System (Applied Biosystems, Carlsbad, CA,United States). The chicken GAPDH gene was used as an internal control. The primers for qPCR are shown in Table 2 [51]. The same cDNA sample (without dilution) was used for all cytokines and GAPDH to normalize and standardize the data. The relative quantification of cytokine gene mRNA was determined via comparison the internal control gene of GAPDH using the $2^{-\triangle \Delta C T}$ method as previously described [52]. A validation experiment was performed by running a dilution series of the cDNA to evaluate the amplification efficiencies of the cytokine genes and internal control gene [51]. The qPCR efficiencies (E) were calculated using the following formula: $E=10^{-1 / \text { slope }}-1$ [53]. Pfaffl correction was conducted for these qPCR analyses.

\section{Determination of serum antibody level}

Blood samples were collected from the wing vein of each chicken at 1-week intervals for 6-weeks post-booster vaccination. Sera were collected for determining Ea143-3-specific antibody levels through indirect ELISA as previously described [49]. In brief, 96-well microtiter plates (Corning-Costar NY, USA) were coated overnight at $4{ }^{\circ} \mathrm{C}$ with $10 \mu \mathrm{g} / \mathrm{ml}$ E. acervulina sporozoites $(100 \mu \mathrm{l}$ protein solution per well) in $0.05 \mathrm{M}$ carbonate buffer ( $\mathrm{pH}$ 9.6). The plates were washed 3 times with PBST, blocked with 5\% Bovine Serum Albumin (BSA) for $2 \mathrm{~h}$ at $37^{\circ} \mathrm{C}$ and then incubated with chicken serum diluted 1:50 in PBS for $1 \mathrm{~h}$ at $37^{\circ} \mathrm{C}$. A 1: 3000 dilution of horseradish peroxidase-conjugated donkey anti-chicken IgY anti-body (Sigma) in 5\% SMP was added as the secondary antibody $(100 \mathrm{ml} /$ well $)$ to detect bound antibodies, and the plates were again incubated for $1 \mathrm{~h}$ at $37{ }^{\circ} \mathrm{C}$. Finally, the complexes were developed by incubation with 3, 3, 5, 5-tetramethylbenzidine (TMB) for $15 \mathrm{~min}$. The reaction was stopped by adding $50 \mu \mathrm{L}$ of $2 \mathrm{M}$ $\mathrm{H}_{2} \mathrm{SO}_{4}$ to each well, and the absorbance was measured at $450 \mathrm{~nm}\left(\mathrm{OD}_{450}\right)$ using an automated ELISA reader. 
Table 2 Primers used for the quantitative RT-PCR

\begin{tabular}{|c|c|c|c|c|}
\hline RNA target & Primer sequence & Accession NO. & Amplification efficiency (\%) ${ }^{a}$ & Correlation coefficients $\left(\mathrm{r}^{2}\right)$ \\
\hline \multirow[t]{2}{*}{ IFN- $\gamma$} & Forward: 5'-AGCTGACGGTGGACCTATTATT-3' & Y07922 & 99.16 & 0.9976 \\
\hline & Reverse: 5'-GGCTTTGCGCTGGATTC-3' & & & \\
\hline \multirow[t]{2}{*}{$\mathrm{IL}-2$} & Forward: 5'-TCTGGGACCACTGTATGCTCT-3' & AF000631 & 98.53 & 0.9932 \\
\hline & Reverse: 5'-ACACCAGTGGGAAACAGTATCA-3' & & & \\
\hline \multirow[t]{2}{*}{ TNFSF15 } & Forward:5'-CCTGAGTTATTCCAGCAACGCA-3' & NM_001024578 & 98.51 & 0.9992 \\
\hline & Reverse: 5'-ATCCACCAGCTTGATGTCACTAAC-3' & & & \\
\hline \multirow[t]{2}{*}{ IL-17D } & Forward:5'-GCTGCCTCATGGGGATCTITGGTG-3' & EF570583 & 98.18 & 0.9954 \\
\hline & Reverse: 5'-CGATGACGGCTTGTTCTGGTTGAC-3' & & & \\
\hline \multirow[t]{2}{*}{ TGF- $\beta 4$} & Forward: 5'-CGGGACGGATGAGAAGAAC-3' & M31160 & 97.39 & 0.9981 \\
\hline & Reverse: 5'-CGGCCCACGTAGTAAATGAT-3' & & & \\
\hline \multirow[t]{2}{*}{$\mathrm{IL}-4$} & Forward: 5'-ACCCAGGGCATCCAGAAG-3' & AJ621735 & 99.41 & 0.9996 \\
\hline & Reverse: 5'-CAGTGCCGGCAAGAAGTT-3' & & & \\
\hline \multirow[t]{2}{*}{ GAPDH } & Forward: 5'-GGTGGTGCTAAGCGTGTTAT-3' & K01458 & 95.48 & 0.9994 \\
\hline & Reverse: 5'-ACCTCTGTCATCTCTCCACA-3' & & & \\
\hline
\end{tabular}

\section{Evaluation of immune protection}

At 14 days of age, chickens were weighed and randomly divided into 13 groups with 30 chickens per group. Experimental group chickens were immunized with $100 \mu \mathrm{g}$ of pVAX-Ea14-3-3 via intramuscular injection in thigh region. The challenged control group and unchallenged control chickens were injected with sterile PBS. The empty vector control group was immunized with $100 \mu \mathrm{g}$ pVAX1 as mentioned above. A booster immunization was given at 7 day after the first immunization. At 28 days of age, the chickens were challenged with freshly sporulated oocyst of E. tenella (5 $\times 10^{4} /$ chicken), E. acervulina $\left(1 \times 10^{5} /\right.$ chicken $)$ and $E$. maxima $\left(1 \times 10^{5} /\right.$ chicken $)$ and mixed sporulated oocysts $\left(5 \times 10^{4}\right.$ E. tenella /chicken, $1 \times 10^{5}$ E. acervulina /chicken, $1 \times 10^{5} \quad$ E. maxima /chicken) separately except the non-immunized and non-challenged group [54]. Six days post-challenged infection, all the chickens were slaughtered. Average body weight gain, oocyst decrease ratio, lesion score, and anti-coccidial index (ACI) were calculated.

The protective efficacy was evaluated based on body weight gain, lesion score, oocyst output, oocyst decrease ratio and ACI $[55,56]$. Body weight gain was determined by weighing the chickens at the end of the experiment and deducing the weight of the same chickens at the time of challenge. Lesion scores were observed and recorded consistent with the method described by Reid and Johnson [57]. The intestinal contents from the whole guts of chickens in all groups were collected and oocysts per gram of content (OPG) were determined via McMaster's counting technique. $\mathrm{ACI}$ is a comprehensive index for assessing the protective effect of immune protection and is calculated as follow: (survival rate + relative rate of weight gain) - (lesion value + oocyst value). According to McManus [58], an ACI $\geq 180$ is considered as high performance, an ACI between 160 and 179 is considered effective, while value of ACI $<160$ is considered ineffective. All the chickens in this study were euthanized by $\mathrm{CO}_{2}$ inhalation. Briefly, $\mathrm{CO}_{2}$ was delivered from compressed gas canister with flowmeter and pressure regulator. After opening the switch, $\mathrm{CO}_{2}$ would be steadily flowing into the euthanasia chamber. The number of animals put in the euthanasia chamber depended on the size of the box to avoid crowding. The entire body of the animal must go into the euthanasia chamber. The gas was delivered with a displacement rate of $20 \%$ of the euthanasia chamber volume per minute. After the animal loses consciousness, a secondary physical method of euthanasia of cervical dislocation was performed.

\section{Statistical analysis}

Non-normally distributed ANOVA with Tamhane's T2 multiple range tests were applied for the determination of statistical significance through SPSS statistical package (SPSS Inc., Chicago, IL, USA). The differences between all groups were tested and $p<0.05$ value was considered as to indicate a significant difference.

\section{Additional files}

Additional file 1: Figure S1. Open reading frames (ORFs) and deduced amino acid sequence of common antigen 14-3-3. (TIF $12897 \mathrm{~kb}$ )

Additional file 2: Table S1. Amino acid similarities of 14-3-3 between E. acervulina, E. maxima, E. tenella, E. necatrix (\%). 1.Ea14-3-3 $=14-3-3$ of $E$ acervulina; Em14-3-3 = 14-3-3 of E. maxima; Et14-3-3 = 14-3-3 of E. tenella; En 14-3-3 = 14-3-3 of E. necatrix. (DOCX 16 kb)

Additional file 3: Figure S2. Identification of recombinant plasmid pVAX-Ea14-3-3 digested by BamH I/Xho I. M: DNA molecular weight marker DL 2000. Lane 1: pVAX-Ea14-3-3. Lane 2: pVAX-Ea14-3-3 digested by BamH I/Xho I. (TIF $62 \mathrm{~kb}$ ) 


\section{Abbreviations}

ACl: Anti-coccidial index; E. acervulina: Eimeria acervulina; E. maxima: Eimeria maxima; E. tenella: Eimeria tenella; Ea14-3-3: Eimeria acervulina 14-3-3 protein; FITC: Fluorescein isothiocyanate; ORF: Open reading frame; PE: Pphycoerythrin; qPCR: quantitative real-time PCR; RT-PCR: transcriptionpolymerase chain reaction

\section{Acknowledgments}

We gratefully thank Jianmei Huang, Zhouyang Zhou for sample collection and valuable suggestions. We also gratefully thank Mr. Muhammad Haseeb and Muhammad Waqqas Hasan for their carful polish in the language of the manuscript

\section{Fundings}

This work was supported by the National Key R\&D Program of China (Grant No. 2017YFD0500401), the National Natural Science Foundation of China (Grant No. 31672545), the Fundamental Research Funds for the Central Universities (Grant No. KYZ201631), the Natural Science Foundation of Jiangsu Province of China (Grant No. BK20161442) and the Priority Academic Program Development of Jiangsu Higher Education Institutions (PAPD).

\section{Availability of data and materials}

The datasets during the current study available from the corresponding author on reasonable request.

\section{Authors' contributions}

SXK designed the study and critically revised the manuscript. LXR, YRF and XLX helped in the study design and analyzed the data. LJH contributed to the main experiment and wrote the draft. LLR performed the laboratory tests. LLJ, TD and LWY contributed to the effective protection experiment. All authors read and approved the final manuscript.

\section{Ethics approval and consent to participate}

Animal experiments were approved by the Institutional Animal Care and Use Committee of Nanjing Agricultural University (approval number: 2012CB120762).

\section{Consent for publication}

Not applicable.

\section{Competing interests}

The authors declare that they have no competing interests.

\section{Publisher's Note}

Springer Nature remains neutral with regard to jurisdictional claims in published maps and institutional affiliations.

\section{Author details}

${ }^{1} \mathrm{MOE}$ Joint International Research Laboratory of Animal Health and Food Safety, College of Veterinary Medicine, Nanjing Agricultural University, Nanjing 210095, People's Republic of China. ${ }^{2}$ Henan Muxiang Veterinary Pharmaceutical Co., Itd, Zhengzhou 450000, People's Republic of China.

Received: 22 January 2018 Accepted: 23 October 2018 Published online: 12 November 2018

\section{References}

1. Williams R. Anticoccidial vaccines for broiler chickens: pathways to success Avian pathol. 2002;31(4):317-53.

2. Morris G, Gasser R. Biotechnological advances in the diagnosis of avian coccidiosis and the analysis of genetic variation in Eimeria. Biotechnol Adv. 2006;24(6):590-603.

3. Blake DP, Tomley FM. Securing poultry production from the ever-present Eimeria challenge. Trends Parasitol. 2014;30(1):12-9.

4. Vermeulen A. Progress in recombinant vaccine development against coccidiosis a review and prospects into the next millennium. Int J Parasitol. 1998:28(7):1121-30.

5. Clarke L, Fodey TL, Crooks SR, Moloney M, O'Mahony J, Delahaut P, O'Kennedy R, Danaher M. A review of coccidiostats and the analysis of their residues in meat and other food. Meat Sci. 2014;97(3):358-74.

6. Meunier M, Chemaly M, Dory D. DNA vaccination of poultry: the current status in 2015. Vaccine. 2016;34(2):202-11.
7. Reid AJ, Blake DP, Ansari HR, Billington K, Browne HP, Bryant J, Dunn M, Hung SS, Kawahara F, Miranda-Saavedra D. Genomic analysis of the causative agents of coccidiosis in domestic chickens. Genome Res. 2014; 24(10):1676-85.

8. You M-J. The comparative analysis of infection pattern and oocyst output in Eimeria tenella, E. maxima and E. acervulina in young broiler chicken.[J]. Vet World. 2014;7(7):542-47.

9. Awais MM, Akhtar M, labal Z, Muhammad F, Anwar MI. Seasonal prevalence of coccidiosis in industrial broiler chickens in Faisalabad, Punjab, Pakistan. Trop Anim Health Prod. 2012;44(2):323-8.

10. Del Cacho E, Gallego M, Lee SH, Lillehoj HS, Quilez J, Lillehoj EP, SánchezAcedo C. Induction of protective immunity against Eimeria tenella, Eimeria maxima, and Eimeria acervulina infections using dendritic cell-derived exosomes. Infect Immun. 2012;80(5):1909-16.

11. Talebi A. Protein profiles of five avian Eimeria species. Avian Pathol. 1995: 24(4):731-5

12. Sasai K, Lillehoj HS, Hemphill A, Matsuda H, Hanioka Y, Fukata T, Baba E, Arakawa A. A chicken anti-conoid monoclonal antibody identifies a common epitope which is present on motile stages of Eimeria, Neospora, and Toxoplasma. J Parasitol. 1998;84(3):654-6.

13. Constantinoiu C, Lillehoj H, Matsubayashi M, Hosoda Y, Tani H, Matsuda H, Sasai K, Baba E. Analysis of cross-reactivity of five new chicken monoclonal antibodies which recognize the apical complex of Eimeria using confocal laser immunofluorescence assay. Vet Parasitol. 2003;118(1):29-35.

14. Liu L, Huang $X$, Liu J, Li W, Ji Y, Tian D, Tian L, Yang X, Xu L, Yan R. Identification of common immunodominant antigens of Eimeria tenella, Eimeria acervulina and Eimeria maxima by immunoproteomic analysis. Oncotarget. 2017:8(21):34935.

15. Cau Y, Valensin D, Mori M, Draghi S, Botta M. Structure, function, involvement in diseases and targeting of 14-3-3 proteins: an update. Curr Med Chem. 2017;24(999):1

16. Lalle M, Curra C, Ciccarone F, Pace T, Cecchetti S, Fantozzi L, Ay B, Breton $\mathrm{CB}$, Ponzi M. Dematin, a component of the erythrocyte membrane skeleton, is internalized by the malaria parasite and associates with Plasmodium 14-33. J Biol Chem. 2011;286(2):1227-36.

17. Zhao N, Gong P, Cheng B, Li J, Yang Z, Li H, Yang J, Zhang G, Zhang X. Eimeria tenella: 14-3-3 protein interacts with telomerase. Parasitol Res. 2014; 113(10):3885-9.

18. Inoue M, Nakamura Y, Yasuda K, Yasaka N, Hara T, Schnaufer A, Stuart K, Fukuma T. The 14-3-3 proteins of Trypanosoma brucei function in motility, cytokinesis, and cell cycle. J Biol Chem. 2005;280(14):14085-96.

19. Assossou O, Besson F, Rouault JP, Persat F, Ferrandiz J, Mayencon M, Peyron F, Picot S. Characterization of an excreted/secreted antigen form of 14-3-3 protein in Toxoplasma gondii tachyzoites. FEMS Microbiol Lett. 2004;234(1):19-25.

20. Meng M, He S, Zhao G, Bai Y, Zhou H, Cong H, Lu G, Zhao Q, Zhu X-Q. Evaluation of protective immune responses induced by DNA vaccines encoding Toxoplasma gondii surface antigen 1 (SAG1) and 14-3-3 protein in BALB/c mice. Parasit Vectors. 2012;5(1):273

21. Schechtman D, Tarrab-Hazdai R, Arnon R. The 14-3-3 protein as a vaccine candidate against schistosomiasis. Parasite Immunol. 2001;23(4):213-7.

22. Yang J, Zhu W, Huang J, Wang X, Sun X, Zhan B, Zhu X. Partially protective immunity induced by the 14-3-3 protein from Trichinella spiralis. Vet Parasitol. 2016:231:63-8.

23. Siles-Lucas M, Merli M, Gottstein B. 14-3-3 proteins in Echinococcus: their role and potential as protective antigens. Exp Parasitol. 2008;119(4):516-23.

24. Shirley MW, Smith AL, Tomley FM. The biology of avian Eimeria with an emphasis on their control by vaccination. Adv Parasitol. 2005:60:285-330.

25. Carvalho FS, Wenceslau AA, Teixeira M, Matos Carneiro JA, Melo ADB, Albuquerque GR. Diagnosis of Eimeria species using traditional and molecular methods in field studies. Vet Parasitol. 2011:176(2):95-100.

26. Ogedengbe JD, Hunter DB, Barta JR. Molecular identification of Eimeria species infecting market-age meat chickens in commercial flocks in Ontario. Vet Parasitol. 2011;178(3):350-4.

27. Chapman HD. Milestones in avian coccidiosis research: a review. Poult Sci. 2014;93(3):501-11

28. Bessay $M$, Le Vern $Y$, Kerboeuf $D$, Yvore $P$, Quéré $P$. Changes in intestinal intra-epithelial and systemic T-cell subpopulations after an Eimeria infection in chickens: comparative study between E. acervulina and E. tenella. Vet Res. 1996;27(4-5):503-14

29. Min W, Lillehoj HS, Burnside J, Weining KC, Staeheli P, Zhu JJ. Adjuvant effects of IL-1 beta, IL-2, IL-8, IL-15, IFN-alpha, IFN-gamma TGF-beta4 and 
lymphotactin on DNA vaccination against Eimeria acervulina. Vaccine. 2001; 20(1-2):267-74.

30. Dlloul RA, Lillehoj HS. Poultry coccidiosis: recent advancements in control measures and vaccine development. Expert Rev Vaccines. 2006;5(1):143-63.

31. Rose ME, Wakelin D, Hesketh P. Interferon-gamma-mediated effects upon immunity to coccidial infections in the mouse. Parasite Immunol. 1991;13(1):63-74.

32. Lillehoj HS, Choi KD. Recombinant chicken interferon- $\psi$-mediated inhibition of Eimeria tenella development in vitro and reduction of oocyst production and body weight loss following Eimeria acervulina challenge infection. Avian Dis. 1998:42(2):307-14.

33. Jang SI, Lillehoj HS, Lee SH, et al. Eimeria maxima recombinant Gam82 gametocyte antigen vaccine protects against coccidiosis and augments humoral and cell-mediated immunity. Vaccine. 2010;28(17):2980-5.

34. Song $X$, Huang $X$, Yan $R, X u L$, Li X. Efficacy of chimeric DNA vaccines encoding Eimeria tenella 5401 and chicken IFN-gamma or IL-2 against coccidiosis in chickens. Exp Parasitol. 2015;156:19-25.

35. Inagaki-Ohara K, Dewi FN, Hisaeda H, Smith AL, Jimi F, Miyahira M, AbdelAleem AS, Horii Y, Nawa Y. Intestinal intraepithelial lymphocytes sustain the epithelial barrier function against Eimeria vermiformis infection. Infect Immun. 2006;74(9):5292-301.

36. Kim W H, Jeong J, Park A R, et al. Chicken IL-17F: Identification and comparative expression analysis in Eimeria- infected chickens[]]. Dev Comp Immunol. 2012;38(3):401-9.

37. Zhao GH, Cheng WY, Wang W, et al. The expression dynamics of IL-17 and Th17 response relative cytokines in the trachea and spleen of chickens after infection with Cryptosporidium baileyi. Parasit Vectors. 2014;7(1):1-7.

38. Strober W, Kelsall B, Fuss I, Marth T, Ludviksson B, Ehrhardt R, Neurath M. Reciprocal IFN-gamma and TGF-beta responses regulate the occurrence of mucosal inflammation. Immunol Today. 1997;18(2):61-4.

39. Jakowlew SB, Mathias A, Lillehoj HS. Transforming growth factor-beta isoforms in the developing chicken intestine and spleen: increase in transforming growth factor-beta 4 with coccidia infection. Vet Immunol Immunopathol. 1997;55(4):321-39.

40. Shalaby MR, Aggarwal BB, Rinderknecht $\mathrm{E}$, et al. Activation of human polymorphonuclear neutrophil functions by interferon-gamma and tumor necrosis factors. J Immunol. 1985;135(3):2069.

41. Park SS, Lillehoj HS, Hong YH, Lee SH. Functional characterization of tumor necrosis factor superfamily 15 (TNFSF15) induced by lipopolysaccharides and Eimeria infection. Dev Comp Immunol. 2007;31(9):934-44.

42. Ivory C, Chadee K. DNA vaccines: designing strategies against parasitic infections. Genet Vaccines Ther. 2004;2(1):17.

43. Lillehoj H, Min W, Dalloul R. Recent progress on the cytokine regulation of intestinal immune responses to Eimeria. Poultry Sci. 2004;83(4):611-23.

44. Awais MM, Akhtar M, Anwar MI, Khaliq K. Evaluation of Saccharum officinarum L. bagasse-derived polysaccharides as native immunomodulatory and anticoccidial agents in broilers. Vet Parasitol. 2018;249:74-81.

45. Song $X, X u L$, Yan $R$, Huang $X$, Shah MAA, Li X. The optimal immunization procedure of DNA vaccine pcDNA-TA4-IL-2 of Eimeria tenella and its crossimmunity to Eimeria necatrix and Eimeria acervulina. Vet Parasitol. 2009; 159(1):30-6.

46. Tomley F. Techniques for isolation and characterization of apical organelles from Eimeria tenella Sporozoites. Methods. 1997;13(2):171-6.

47. Sambrook J, Russell DW. Molecular cloning: a laboratory manual[M]. Cold Spring Harbor Laboratory. 2001;675.

48. Song H, Qiu B, Yan R, et al. The protective efficacy of chimeric SO7/L-2 DNA vaccine against coccidiosis in chickens. Res Vet Sci. 2013;94(3):562-7.

49. Song $X$, Zhao $X, X u L$, Yan R, Li X. Immune protection duration and efficacy stability of DNA vaccine encoding Eimeria tenella TA4 and chicken IL-2 against coccidiosis. Res Vet Sci. 2017;111:31-5.

50. Sasai K, Aita M, Lillehoj H, Miyamoto T, Fukata T, Baba E. Dynamics of lymphocyte subpopulation changes in the cecal tonsils of chickens infected with Salmonella enteritidis. Vet Microbiol. 2000;74(4):345-51.

51. Song H, Song X, Xu L, Yan R, Shah MAA, Li X. Changes of cytokines and IgG antibody in chickens vaccinated with DNA vaccines encoding Eimeria acervulina lactate dehydrogenase. Vet Parasitol. 2010;173(3):219-27.

52. Livak KJ, Schmittgen TD. Analysis of relative gene expression data using real-time quantitative PCR and the $2^{-\Delta \Delta C T}$ method. Methods. 2001;25(4): 402-8.

53. Sanchez H, Chapot R, Banzet S, Koulmann N, Birot O, Bigard AX, Peinnequin A. Quantification by real-time PCR of developmental and adult myosin mRNA in rat muscles. Biochem Biophys Res Commun. 2006;340(1):165-74.
54. Holdsworth PA, Conway DP, Mckenzie ME, Dayton AD, Chapman HD, Mathis GF, Skinner JT, Mundt HC, Williams RB. World Association for the Advancement of veterinary parasitology (WAAVP) guidelines for evaluating the efficacy of anticoccidial drugs in chickens and turkeys. Vet Parasitol. 2004;121(3-4):189.

55. Morehouse NF, Baron RR. Coccidiosis: evaluation of coccidiostats by mortality, weight gains, and fecal scores. Exp Parasitol. 1970;28(1):25-9.

56. Chapman H, Shirley M. Sensitivity of field isolates of Eimeria species to monensin and lasalocid in the chicken. Res Vet Sci. 1989;46(1):114-7.

57. Johnson J, Reid WM. Anticoccidial drugs: lesion scoring techniques in battery and floor-pen experiments with chickens. Exp Parasitol. 1970;28(1):30-6.

58. McManus EC, Campbell WC, Cuckler AC. Development of resistance to quinoline coccidiostats under field and laboratory conditions. Int J Parasitol. 1968;54(6):1190-3.

\section{Ready to submit your research? Choose BMC and benefit from:}

- fast, convenient online submission

- thorough peer review by experienced researchers in your field

- rapid publication on acceptance

- support for research data, including large and complex data types

- gold Open Access which fosters wider collaboration and increased citations

- maximum visibility for your research: over $100 \mathrm{M}$ website views per year

At BMC, research is always in progress.

Learn more biomedcentral.com/submissions 